\title{
Discussão sobre a Problemática na Interface entre as Funções Marketing e Produção
}

\section{Denise Ávila de Melo}

Doutoranda da Escola de Administração de Empresas da Universidade de Munique Hedivig-Dransfeld-Allee 24

80637 Munich - Alemanha.

email: DA051263@highlander.cip.bwl.uni-muenchen.DE .

Palavras-chave : funções industriais, marketing, produção, integração

Key-words : Inclustrial finctions, marketing, production, integration

\section{RESUMO}

$\dot{E}$ comum se encontrar em uma empresa industrial, divisões e/ou departamentos com funçōes bastante especificas o que muitas vezes resulta em conflitos. Neste trabalho, será discutida a impontância de haver uma boa integração entre duas das mais importantes funções de uma empresa industrial, quais sejam : marketing e produção. Também serão discutidas as principais áreas de conflito entre estas duas funções e as causas de tais conflitos. Pretende-se acima de tudo salientar que as razões dos conflitos, apesar de complexas, podem ser entendidas, e mais ainda, que os mesmos podem ser atenuados. como por exemplo, por meio de programas de fomento a cooperação entre as fiunções.

\begin{abstract}
It is usual to find among industrial enterprises, departments and divisions with specific functions, sometimes resulting in conflicts. The importance of providing good integration between two of the most important functions. within an industrial enterprise. production and marketing, is discussed in this paper. We also discuss the main conflict areas between these two functions and the reasons for that. The basic purpose is to emphasize that even complexes, the reasons can be understood and minimized.
\end{abstract}




\section{Introdução}

É comum se encontrar em uma empresa industrial divisões distintas da empresa, especializadas de acordo com as funções que exercem. Assim funções como marketing e produção são normalmente exercidas por divisões ou departamentos separados, neste caso denominados divisão ou departamento de marketing e produção. De todas as divisões de uma empresa, marketing e produção são as mais imporlantes, não se querendo menosprezar, porém, o valor de outras divisões como por exemplo: desenvolvimento de produtos, planejamento, finanças, contabilidade $\mathrm{e}$ controlc, administração ctc. Estas, todavia, de uma forma ou de outra dependem das outras duas. Uma boa interface entre marketing c produção ć portanto essencial para uma cmpresa industrial. Entretanto, conflitos cntre cssas duas divisões são muito comuns.

Será ressaltado neste trabalho a importância de haver uma boa integração entre as funções marketing c produção, as principais áreas de conflito entre cstas duas funções $\mathrm{c}$ as causas de tais conflitos. Pretende-sc, acima de ludo, salientar que as razões dos conflitos, apesar de complexas, podem ser entendidas, c mais ainda, que os mesmos podem ser atenuados, como por cxcmplo, por meio de programas de fomento à cooperação cutre as funções. Embora muitos dos problemas analisados aqui possam ser deteclados na interface com e cntre outras funções tambćm, um cxcmplo é a interface cntre marketing $\mathrm{e}$ descivolvimento de produto, ênfase serí dada somente a marketing e produção.

\section{O Porquê de se Entender os Conflitos e Tentar Resolvê-los}

Segundo Michael Porter (1989), atividades criadoras de valores (key valuc-adding activities) como: logistica interna, operações, marketing c vendas, logística externa e serviço ao consumidor, assumem um papel chave na criação de vantagem competiliva para uma empresa. Na maioria das empresas industriais estas atividades são excrcidas ou por marketing ou pela produção.

Em uma empresa industrial ć a produção que cria os valores que pagam por tudo c todos no negócio. Ela agrega todas as outras funções da empresa em volta de si. A produção, porém, pode ser uma arma competiva em vez de apenas uma simples fonte de recursos para gerar os produtos da empresa (WHEELWRIGHT 1984). A produção pode e deve ser capaz de ajudar a empresa a conseguir alcançar seus objetivos sem desperdício de recursos com atividades de baixa prioridade ou que não criam valor para os consumidores. A tarefa da produção ć a de prover, melhor do que seus competidores, os critérios que irão permitir que os produtos da cmpresal consigam uma maior participação no mercado.

A divisĩo de Marketing, por sua vez, ć que define segmentos de mercado que serão alvo, traduz cxigências do consumidor $\mathrm{cm}$ termos de produto e volume de vendas, 
decide sobre a linha de produtos c estabelece políticas de preço, promoção de vendas e serviço ao consumidor.

A gerência da produção toma decisões quanto a cxpansão da capacidade, layout $\mathrm{e}$ localização das facilidades produtivas, quanto a tipo de iccnologia a ser empregada no processo de produção $\mathrm{e} d \mathrm{de}$ controle de qualidade assim como quanto a politicas de plancjamento da produção, estoque, aquisição de matcrial c mão-de-obra. Decisões sobre layout c localização da facilidade produtiva assim como sobre o tipo de tecnologia a ser usado no processo requerem suposições acerca de volume de produção, linha de produtos, estratcégias competivas tais como baixos custos de produção ou Mlexibilidade, localização do mercado consumidor atual $\mathrm{c}$ de potenciais mercados consumidores. Estas suposições. por sua vez derivalu de informações vindas do consumidor. que são interpreladas $c$ fornecidas por marketing. O plancjamento da produção, o controle de qualidade assim como a política de pessoal dependem de suposições relativas a linha, volume c qualidade de produtos e serviço de entrega. Estas informações tambćm sĩo providas por marketing. Por outro lado, a gerência de marketing depende da produção para poder definir preço. programas promocionais $\mathrm{e} \mathrm{de}$ desenvolvimento da imagem dos produtos, qualidade dos produtos, flexibilidade do processo, politicas de csloque $\mathrm{c}$ plancjamcnto

O que se quer mostrar é que dentro das cmpresas são lomadas uma sćric de decisões que cuvolvem acima de ludo as funções de marketing c produçĩo. Estas decisõcs precisam ser continuamente revisadas $\mathrm{c}$ algumas vezes atć alteradas já que não só os produtos da empresa como também a própria concorrência evoluem $\mathrm{c}$ amadurccem. Michael Porter (1989) é de opinião que, quando as funções de uma cmpresa compartilham uma visão comum $\mathrm{c}$ comprecndom as implicações cstratćgicas de suas decisões, então estas funções $10 \mathrm{mam}$ decisões c atiludes que implementam uma estratégia que gera valor para os consumidores. Hayes et al. (1979) acham que se a coordenação cntre marketing c produção for difícil isto faz. com que a conpresa seja mais vulnerável aos ataques dos competidores. Já uma pesquisa condurida por John el al. (1991) chega mesmo a cvidenciar que consenso entre objclivos c atos de markcting c produção cstá relacionado com o mellor desempenho cm lermos de vendas. Portanto considerivel vantagem competitiva pode acorrer a uma cmpresa capaz de integrar suas funções de marketing c produção com uma estratégia comum. Shapiro (1977) vai mais além ainda afirmando que "a empresa prospcrara quando suals divisõcs de markcling c prodıção opcrarem $\mathrm{cm}$ uma atmosfera de cooperação c passárem a comprecnder que cada uma tem sua função a cxercer $\mathrm{c}$ suas necessidades a satisfazcr, c que nenhuma função pode subverter a outra".

E mais, se cmpresas continuarem a lidar com relações ao consumidor cada vez mais fortes c complexas $\mathrm{c}$ a se esforçar cada vez. mais por maior lucratividade através da especialização de seus produtos. clás terão que descuvolver melhores mecanismos de comunicaçĩo interna (SHAPIRO 1987a). 
Produtos especializados requerem muito mais integração funcional do que produtos que já viraram commodities (SHAPIRO 1987b). Também quanto mais complcxo o mercado c o ambiente onde a cmpresa tem seus negócios, quanto mais flexível $c$ inovativa deve ser a cmpresa (RUEKERT et al. 1987). Estas situações criam maior necessidade de várias áreas da empresa terem que interagir entre si e serem muito mais capazes de tomarem decisões conjuntas para o melhor interesse de longo prazo da empresa como um todo.

\section{Áreas Problema e Sugestões para Solucionar os Conflitos}

Shapiro (1977) identifica as seguintes árcas onde a cooperação entre as duas funções é requerida c conflitos ocorrem com mais frequência: 1) plancjamento da capacidade produtiva $\mathrm{c}$ previsão de vendas a longo prazo; 2) plancjamento da produção c previsão de vendas a curto prazo; 3) entrega c distribuição física; 4) qualidade; 5) diversidade da linha de produtos: 6) controle de custos; 7) lançamento de novos produtos; 8) serviços adjuntos. A seguir serão discutidas cada uma destas áreas problema c scrĩo fcilas algumas sugestõcs para se atenuar os conflitos.

\section{Planejamento da Capacidade Produtiva e Previsão de Vendas a Longo Prazo}

O planejamento da capacidade produtiva $\mathrm{c}$ a previsão de vendas a longo prazo cxigem intensa troca de informaçõcs cntre marketing c produção. A produção faz uso da previsão de vendas a longo prazo para planejar a capacidade produtiva. Estas previsões, porćm, não são produto de uma ciência cxata, pelo contrário. clas são cstimativas subjetivas, geralmentc baseadas na intuição do pessoal de vendas, que tem contato direto com o consumidor c que, no cntanto, tende a reagir cmocionalmente $c$ a ser muito pessimista quando o negócio está devagar c muito otimislas quando cle vai bem. Muilas vezes cstas previsões não passam literalmente de um "chute", portanto não se deve esperar que elas sejam livres de crros. E para piorar a siluação, o pessoal da produção costuma revisar a previsão de vendas feita por markeling, apesar de a produçâo ser a função que está mais isolada do mercado c mais afastada dos consumidores, $c$ com base na previsão revisada planejar capacidade produtiva, csloque c contratar cmpregados.

O problema é que se a capacidade produtiva for baisa para cobrir a demanda real, a cmpresa perderá negócio e marketing colocará a culpa na produção. Dai surge a necessidade de se construir estoques de segurança, cujos custos recaem sobre a produção. Pior ainda é quando estes estoques de segurança acabam se transformando cm anos de cstoque de produtos que nunca mais ou dificilmente scrão vendidos. Excesso de capacidade produtiva támbém sai a altos custos para a produção, o que por sua vez se reflete no preço final do produto. Em resumo, estes problcmas afctam muito mais a produção.

O ideal mesmo seria ter a capacidade 
produtiva neccssária para cobrir a demanda, mas a pergunta ć: como? É neccssário tempo para se conseguir alterar a capacidade produtiva de uma empresa, principalmente quando o aumento de capacidade requer novas instalações industriais, novos equipamentos $c$ mais funcionários, que além do mais precisam ser treinados. É mais barato e mais rápido investir na melhoria das previsões de vendas a longo prazo, tomando-as mais precisas. Não obstante não se possa cxcluir de lodo incerlezas nas previsões de demanda a longo prazo, há pelo menos formas de se melhor gerenciá-las. Uma delas é sugerida por Fisher et al. (1994). Estes afirmam, que a partir de uma análise do histórico de vendas, é possível distinguir produtos, cujas vendas são mais previsiveis, daqueles, cujas vendas não são, c assim programar uma produção antccipada dos primeiros c uma produção reativa dos últimos. Uma outra sugestão é cstimular a troca continua de informações $c$ o traballo conjunto entre marketing $c$ produção neste caso, pois quanto mais cedo a produção for informada de mudanças na previsĩo, mais tempo cla terí para reagir. Assim, por cxemplo, pode-se fazer com que as duas divisões decidam juntas sobre questões como linha de produtos, tipo de tecnologia utilizada no processo industrial, clc.. Também se pode traballar no scntindo de diminuir o leat-time da cadcia produtiva, desde o da aquisição de matéria prima atć o do processo produtivo, pois quanto menor o lead-time maior a capacidade de reação da produção a mudanças nas vendas. Devemll-sc também tentar assinar contratos mais flexiveis com os fornecedores, principalmente no que di\%. respeito a mudanças nas cspecificações de quantidades.

\section{Planejamento da Produção e Previsão de Vendas a Curto-Prazo}

Trata-se de uma versão a curto prazo do problema anterior. Mais uma vez surgem conflitos porque a previsão de vendas não ć cxata c o plancjamento da produção não ć $100 \%$ flexível. Além do mais, os interesses do gerente da produção $\mathrm{c}$ os do gerente de vendas são quase que oposios neste caso. $O$ gerentc de produção quer otimizar a utilização das máquinas, maximizar a produção rodando lotes grandes, minimizar os custos cvitando frequentes trocas de produtos nas linhas e tambćm manter um número constante $\mathrm{c}$ cstável de funcionários nas mesmas. Enquanto que os gerentes de vendas querem que a produção rode lotes pequenos e mantenla um estoque variado. Eles também querem que os pedidos dos clientes, mesmo que feitos a curtíssimo prazo, scjam atendidos imcdiatamentc. Incentivar a integração entre marketing $c$ produção ć neste caso um fator muito importante, principalmente para que ambas funções cheguem a um consenso que beneficic a cmpresa como um todo. Também podeml-se implantar medidas que tornem as operações da fábrica mais produtivas quando da produção de lotes pequenos. Entre clas se sugerem aqui TOC (Theory of Constraints) (GOLDRATT et al. 1986 c GOLDRATT 1990) c JIT (Jnst-in-T'ime). Muitas vezes também mudanças a curto prazo na programação da produção podem ser dificultadas por falta de materia-prima disponivel, o que acontece principalmente se pedidos de um 
certo produto ou de mais produtos ultrapassarem a previsão de longo prazo $\mathfrak{c}$ o lead-time do fornecedor for grande. Neste caso sugere-se procurar fomecedores que tenham capacidade de reação mais rípida.

\section{Entrega e Distribuição Física}

O interesse principal de marketing ć manter o alto nível de scrviço ao consumidor. Um dos requisitos para isso ć que a empresa entregue os produtos $\mathrm{ma}$ quantidade $\mathrm{c}$ na data que o consumidor os desejar. Muitas vezes, c atualmentc isto tem se tornado uma realidade. uma resposta rípida aos pedidos do consumidor é não só uma questão de vantagem competitiva como também até de sobrevivência da cmpresa. Um cxemplo é o mercado de peças de reposição. Este cxige que a cmpresa mantenha todos scus produtos $\mathrm{em}$ estoque c a um nivel que cubra qualquer demanda. Altos estoques de produtos acabados acarretam altos custos para a produção. A prodıção quer logicamente manter os estoques baixos, mas isto, principalmente quando o lead-fime ć grandc. não ć possível sem que ocorra muitas veres que um produto não cstcja disponivel para venda cxatamente quando o consumidor o descja. Um fator que influi no lead-time ć o tipo de tecnologia utilizada no processo produtivo. Tecnologias de produção que reduzem drasticamente o lead-time, principalmente quando a linha de produtos da cmpresa ć muito vasti, tais como sistemas flcxívcis de manufatura (FMS) ou mesmo CIMS, são cxtremamente caros c o allo nível do serviço ao consumidor requerido por markcting pode não sair a um preço competitivo. Por isso, é muito importante que marketing $\mathrm{c}$ produção interajam na tomada destas decisões.

\section{Qualidade}

Uma outra área de conflitos cstá nas diferentes formas que as funções vêm entender o que seja qualidade para os consumidores. Para o pessoal de marketing os consumidores descjam ter muitas opções, que os produtos scjam variados c possuam características avançadas. Porćm, muitas vezes o pessoal de marketing não sabe que estas "muitas opções" podcm dificultar c muito a tarcfa da produção, podendo tormar o processo produtivo $\mathrm{c}$, consequentemente, o produto muito mais caros. Meticulosidade, sofisticação, qualidade, diversidade de produtos $\mathrm{c}$ caracicristicas tornam o processo produtivo muito complexo. Muita complexidade pode gerar problemas de qualidade no processo de produção ou até mesmo problemas que só aparecerão posteriormente, quando cstes tcrão que ser resolvidos $\mathrm{cm}$ campo. Muilas vezes também marketing quer clevar a qualquer preço a qualidade dos produlos scm cntretanto estar cicnte que às vezes vale mais a pena cconomicamentc para a cmpresa consertareventuais problcmas que possam aparecer depois do produto já vendido, do que se elevar a qualidade ou os padrões de inspeção no processo produtivo. Alćm disso ć muito mais difícil manter o pessoal da produção familiarizado com todos os produtos se as opções forem muilas c ou se a linha de produtos for muito vasła. Pouca familiarização do pessoal da 
produção aumenta as chances de que ocorram falhas na produção. Mais uma vez, cslas decisões precisam ser tomadas conjuntamente por marketing c produção. Uma tomada de decisão unilateral pode ser prejudicial à cmpresa.

\section{Diversidade da Linha de Produtos}

O pessoal de marketing deseja uma vasta linha de produtos. Entrelanto uma linha de produtos muito ampla pode ser muilo cara. Por cxcmplo, cngcnheiros, funcionários da produção c o pessoal de manutenção precisam ser capazes de mancjar eficientemente uma grande varicdade de modelos ou produtos. Isto implica na necessidade de mão de obra mais qualificada ou de um maior número de funcionários especializados $\mathrm{cm}$ alguns poucos produtos. Podc acarretar também perda de capacidade produtiva devido à necessidade de se ter que preparar as máquinas scmpre que se for fabricar um modelo diferentc. A intensa troca de produtos c preparação de máquinas pode causar também stress no pessoal da linha. Aumentam com tum maior numero de ordens pequenas os gastos com processamento, cxpedição $\mathrm{c}$ transporte. Uma vasta linha de produtos pode acarretar confusão para 0 pessoal de vendas e para os distribuidores, que alćm do mais terão de ser capazes de dar suporte aos consumidores de todos os produtos da cmpresa. E o maior de todos os problcmas cstá no nĩo aprovcitamento das vantagens proporcionadas por uma economia de escala. Só que, por outro lado, uma linha de produtos imuito limilada pode implicar $\mathrm{cm}$ perdas nas vendas devido a insatisfação dos consumidores ou ao fato da empresa não cstar representada em algumas áreas $\mathrm{c}$ cm alguns segmentos de mercado. Muitas vezes uma vasta linha de produtos ć uma questão de sobrevivência para a empresa que se encontra em um mercado em declínio. Com isto a empresa pode participar em vários mercados e contrabalançar prejuizos $\mathrm{cm}$ um com ganhos em outro. O problema ć que uma vasta linha de produlos acarreta custos diretamente mensuráveis para a produção $c$ uma estreita, cuslos mensuráveis cm marketing, $\mathrm{c}$ aí se localiza a base para conflitos entre as duas funções. Mais umn vez cstc é um problema que tem que ser resolvido por marketing $\mathrm{c}$ produção juntos.

Caso a linha de produtos scja muito vasta c as características dos produtos scjam muito variadas, principalmentc no que se refere a volume de vendas, pode-se pensar cm uma solução como a do lipo rábricas focadas (SKINNER 1977 c WILDEMANN 1992) que sugcre a instalação de facilidades produtivas cspecializadas por produtos ou por grupo de produtos, que melhor atendem às necessidades produtivas dos mesmos, desde que o volume de produção $\mathrm{cm}$ cada facilidade consiga ser mantido a um nivel competitivo.

\section{Controle de Custos ।}

São os custos que detcrminam os preços 
c os lucros. Scgundo markcting, altos custos são devidos à inépcia da gerência da produção. Para o pessoal da produção altos custos são devidos às cxigências despropositadas do pessoal de markcting, tais como cntrega rípida, alta qualidade, vasta linha de produtos c abusivo lançamento de novos produtos.

\section{Lançamento de Novos Produtos}

$\mathrm{O}$ que o pessoal de marketing muitas vezes considera como uma pequena modificação $\mathrm{cm}$ um produto pode no fim requerer grandes mudanças na área da produção, principalmente se para as modificações são necessários novos processos produtivos. treinamento de funcionários. novos equipamentos $\mathrm{c}$ muitas tentativas e crros até que o equipamento estcja totalmente ajustado ao processo produlivo. Portanto antes de lançar novos produlos é necessário se verificar se os beníficios do lançamento para o consumidor c. consequentemente, para o posicionamento da empresal no mercado compensarão todos os transtonlos que scrão causados.

\section{Serviços Adjuntos}

Frequentemente podem ocorrer conflitos quando alguns serviços como instalação. prestação de serviço cm campo c reparos forem de responsabilidade tanto de markcting quanto da produçĩo. Um problema ć que o pessoal de marketing cucara instalação como uma prestação de scrviço ao consumidor $\mathrm{c}$ a produção como una simples opcração final do processo produtivo.

\section{Entendendo as Causas dos Conflitos.}

Shapiro (1977) distinguc quatro causas básicas para os conflitos: o sistema de avaliação e recompensa, diferenças culturais, na oricntação c na experiência dos funcionários.

Ambos, aqueles que gerenciam uma funçĩo c os scus funcionários, lĉm diferentes interesses, cxperiências e modos de cncarar o mundo. Por isso não ć de sc cspantar que funçõcs tenham problemas com integração. Entre markeling c produção então, cslas diferenças são mais acentuadas ainda. Geralmente, o gerente de markcting comç̧ou sua carrcira cm vendas. onde cle "vivia e morria" pelo consumidor. O gerente da produção começou como lider ou chefe de linha e fe $\%$ carreira através das operaçôes da fábrica.

O gerente de markeling cstá voltado pan os consumidores; seu escritório está perto do de pessoal de vendas e logo ele cstá cxposto aos problcmas do consumidor. $\mathrm{O}$ da produção está voltado para as operações da fábrica, tem mais contato com os funcionários dela c lida mais com os problemas deles. Em resumo. cada gerente cslá muito mais cicnte da situaçĩo $\mathrm{c}$ dos problcmas de sua própria função. Cada um se scnte muito mais a vontade com sua própria função c cstá muito mais afinado com scus próprios subordinados, pois cle é 
quem os contratou e os treinou c ć com cles que cle compartilha suas expericencias $\mathrm{e}$ pontos de vista. Ele cutcnde suas oricntações $\mathrm{c}$ atitudes.

Além disso, cada função de uma cmpresa reage no sentido de proteger scus próprios intcresses, que normalmente consistem tanto na minimização de scus cuslos, maximização de scus benefícios. quanto na avaliação positiva c recompensa apropriada dos scus funcionários. Os interesses da produção podem ser resumidos em querer rodar as linhas da forma mais harmônica e mais cconômica possivel. A produção ć muito mais orientada para custos do que para lucros $\mathrm{c}$ tende a accitar mudanças somente quando cstas inpplicam em significativa redução de custos. Já marketing está mais interessada por $11 \mathrm{~m}$ crescimento lucrativo da compresa através de vendas. participação no mercado e conquista de novos mercados. Marketing. por outro lado, tambćm ć muito mais oricnlada para vendas do que para lucros, $\mathrm{c}$ quer sempre gerar novos produlos. desenvolver novos programas, ctc.

Porque os gerentes têm cxperiências culturais c orientações tão diferentes $\mathrm{c}$ se diferem tanto no jeito de viver assim como no de gerenciar, fica difícil o trabalho $\mathrm{cm}$ conjunto. Logo há um grande potencial para problemas de comunicação entre as duas funções c estas dificuldades podem conduzir a conflitos.

Um problema se encontra também na fonte de dados que as duas funções utilizam. Os dados, dos quais a produção faz uso, são cxtremamente quantitativos $\mathrm{c}$ facilmente mensuráveis como custos de uma cxpansão de capacidade. Já marketing utiliza dados predominantementc qualitativos. Por exemplo, vendas c nivel da utilização da capacidade são difíccis de screm previstos.

\section{Polindo a Interface}

Quanto maior a cmpresa quanto mais difícil se toma o gerenciamento da interface cntre marketing c produção pois há um maior número de pessoas cnvolvidas. $\mathrm{Na}$ verdade, a comunicação se torna cxponencialmente mais dificil quando o numero de pessoas ou gnupos que precisam de se communicar entre si cresce (SHAPIRO 1987b). Muitas vezcs outras funçõcs cntram no quadro $c$ adicionam complexidade a certas áreas de tomada de decisão. Por cxemplo, em muilas empresas markcting c produção $\mathrm{tem}$ que intcragir com a função de desenvolvimento ou engenlaria do produto. Assim. $\mathrm{cm} v \mathrm{cz}$ do duas superfícies de contato ou. possívelmente de atrito. passam a haver quatro. Outro cxemplo é o cnvolvimento do pessoal de finanças cm questões de plancjamento da capacidade produtiva e do pessoal de controle de custos no controle de estoques c custos de distribuição. Por isso todas as superfícies de contato devem ser bem polidas.

Em si não há nada de crrado com o fato das funções quererem defender seus próprios interesses. Muitas vezes isto pode gerar até uma concorrência saudável entre clas. Porém. isto só acontece quando todas 
as funções estão sintonizadas com as cstratćgias da cmpresa. Por isto, a implantação de uma estratćgia unificada da cmpresa mostrando um único conjunto de "ordens para marchar" $c$ de prioridades $c$ mostrando também o papel de cada função dentro deste esquema ć geralmentc o primeiro passo a ser dado no sentido de melhorar a inlerface entre as funções (SHAPIRO 1987a). Com a cxistência de uma cstratégia unificada c cxplicitada cntrc as várias divisões é bem menos provável que por cxcmplo markeling $\mathrm{c}$ produção venham a discutir sobre o que pode ser mais importante para compresa. Ier uma vasta linha de produtos ou rodar a produção da forma mais harmônica possivel, pois cstas prioridades cstarño claras na estratćgia. E durante o processo de definição destas cstratégias as divisões terão que discutir todos aqueles problemas citados no começo. Isto obriga que as funções cheguem a um acordo. Depois, com base na cstratćgia unificada ć que as diversas funções vão descnvolver políticas internas. Se estiver estabelecido nas cstratégias da cmpresa que ela scrí fornecedora de produtos de baixo custo, então, markcting tem que trabalhar no sentido de cooperar com a produção na redução dos custos da mesma. Isto pode implicar por exemplo que o pessoal de vendas só accite ordens de grande volume, ou que sacrifique qualidade por custo, etc.

Uma out ra forma de se gerenciar melhor as interfaces cnt re as funções ć modificando o sistcma de avaliação c recompensa no scntido de incentivar cooperação entre as funções c não apenas competência funcional. Por exemplo. se entrega pontual for uma estratégia cxplícita da empresa. então o pessoal de produção deve ser avaliado não só $\mathrm{cm}$ termos de custos. como tambćm cm termos de pontualidade na entrega c no tempo de resposta do sistema produlivo.

Já que as pessoas tendem a trabalhar mais $\mathrm{cm}$ colaboração com aquelas que lềm escritórios perto dos scus, uma forma de encorajar cooperação interfuncional ć transferir os escritórios daqueles que precisam de trabalhar em cooperação para perto um do outro. O uso de sistemas de informação tais como correio cletrônico ajuda a diminuir os problemas de comunicação c a "cncurtar" as distâncias geográficas permitindo que o pessoal dentro da organização se comunique mais facilmente. Embora estes sistemas de comunicação nunca venlam a substituir a importância de um contato pessoal, cles têm a vantagem de serem mais informais e de quebrarem as barreiras hierárquicas.

Implementação de planos de carreira interfuncional ajuda aos gerentes terem um melhor entendimento dos problemas das outras funções. Todavia, não se deve cxagerar c nem ć sempre que um plano de carreira interfuncional pode ou deve ser implementado, principalmente $\mathrm{cm}$ empresas onde especialização funcional ć um fator competitivo. Razoável scria que todo gerente tivesse experiência cm pelo menos duas funções $\mathrm{c}$ uma boa noção de como as demais traballam.

Acima de tudo, o apoio da direçĩo da cmpresa é muito importante. Ela deve 
cstimular a cooperação entre as funçõcs também nos altos niveis hicrárquicos $c$ incentivar que problemas comuns a duas funçõcs ou divisões scjan resolvidos cntrc elas mesmas.

\section{Conclusão}

Este texto fornerceu uma visão geral sobre a problemática da interface cntre as funçõcs markcting c produção. Foi mostrado que os problemas envolvendo conflitos cutre marketing $\mathrm{c}$ produção são reais c importantes c que uma boa coordenação cutre as duas funçõcs ć uma questão de vantagem compctitiva. Conflitos entre divisões podem ocorrer porque as funções c pessoas que nelas trabalham são difcrentcs, têm cxperiências c orientações diversas e sempre agem no sentido de proteger seus próprios interesses. Porém, por mais complexos que os conflitos entre as funções possam ser, há sempre formas de icntar contorná-los, amcnizá-los ou mesmo clcminá-los. Mas o mais importante ć que a empresa tenla bem definida suas estratégias de mercado. para que assim se consiga consenso não só entre marketing c produção mas também cntrc todas as funções.

\section{Bibliografia}

CRITTENDEN, V. L. Close the Markcting/Manufacturing Gap. Sloan Management Revicw, Spring 1992, +1-52.

DRUCKER, P. F. The Emerging
Theory of Manufacturing. Harvard Business Review, May-June 1990, 94-1(02.

FISHER, M. L., HAMMOND, J. H., OBERMEYER, W. R. \& RAMAN, A. Making Supply Mect Damand in an Uncertain World. Harvard Business Rcviciv, May-Junc 1994, 83-93.

GOLDRATT, E. M. \& COX. J. The Goal: A Process of Ongoing Improvement. Spectnum, New Haven. Comm., 1986.

GOLDRATT. E. M. What is this thing called Theory of Constraints and how it should be implemented? North River Press, Inc., Croton-on-Hudson, N.Y., 1990.

HAYES, R. H. AND WHEELWRIGHT, S. C. Link manufacluring process and product life cycles. Harvard Business Review, January-Fcbruary 1979, 133-140.

JOHN, C. H. ST, \& RUE, L. W. Rescarch notes and communications co-ordinating mechanisms, consensus between marketing and manufacturing groups. and marketplace performance. Strategic Management Journal, Vol. 12. $1991,549-555$.

MILLER, J. G. Fit production systems to the task. Harvard Business Revicu, January-February 1981, 145-154.

PORTER, M. E. Compctitive Advantage. Campus Verlag. New York, 1989.

PORTEUS. E. L. \& WHANG, S. On 
Manufacturing/Marketing Incentives. Marketing Science, Vol. 37, Scptember 1991, 1166-1191.

RUEKERT, R. W. \& WALKER JR., O. C. Marketing's Interaction with Other Functional Units: A Conceptual Framework and Empirical Evidence. Journal of Marketing. Vol. 51 (January 1987), $1-19$.

SHAPIRO, B. P. Can Markcling and Manufacluring Cocvist? Hanard Business Revicw, September-October 1977, 104-114.

SHAPIRO, B. P. The New Intimacy. Harvard Business School, class discussion note No. 9-587-121, 1987a.

SHAPIRO, B. P. Functional Integration: Getting All The Troops To Work Together. Harvard Business School, class discussion notc No. 9-587-122. $1987 \mathrm{~b}$.

SHAPIRO, B. P. Varicly Versus Valuc: Two Generic Approaches to Product Policy. Harvard Busincss School, class discussion notc No. 9-587-119, 1987c.
SHAPIRO, B. P. The Magic Matrix: Products and Accounts. Harvard Business School, class discussion note No. 9-588-006, 1987d.

SHAPIRO, B. P. What the Hell Is "Market Oricnted". Harvard Business Revicw, November-December 1988.

SHAPIRO, B. P., RANGAN V. K, \& SVIOKA, J. J. - Staple Yourself to an Order. Harvard Busincss Revicw, July-Augus1 1992, 113-122.

SKINNER, W. The focused factory. Harvard Business Review, May-Junc 1974, $113-131$.

WHEELWRIGHT, S. C. Manufacturing Strategy: Defining the Missing Link. Strategic Management Journal, Vol. 5, 1984, 77-91.

WILDEMANN, H. Dic modulare Fabrik - kundennahe Produktion durch Fertigungssegmentienung. gmft, St. Gallen, 1992

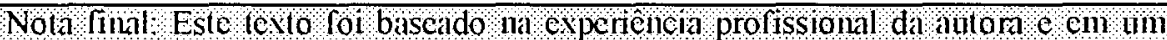
levantanento bibliografico feilo pela liesma cni preparaço para una palestra com mesino lítilo aprescillada en un seminário na Escola de A dministraçà de Enupresás da Universidade de Munique (Fakulat fit Belriebswirtschaftleltre der Liduvig-Mixinilians-Universita Müchen), Munique, Alemanila:

A gradecimenios. A antora igradece imensamenle Prof Benson P Shapiro da Escola de Negocios da Universidade de Hanard (Harvard Business School) Boslon. EU A por ter gentilmente cedido parte do nialenial de referéncia para esie traballio. inclisive sias anotaçócs parn discussî́ eli aula. 